\title{
Análise morfológica de nanofibras \\ Uma abordagem por visão computacional e aprendizagem de máquina
}

\author{
Guilherme Duarte de Barros \\ Escola de Engenharia, Arquitetura e \\ Tecnologia \\ Universidade Anhembi Morumbi \\ São Paulo, SP - Brasil \\ guilherme.barros@anhembi.br
}

\author{
Wesley da Silva Pereira \\ Escola de Engenharia, Arquitetura e \\ Tecnologia \\ Universidade Anhembi Morumbi \\ São Paulo, SP - Brasil \\ wes.ccbpereira@gmail.com
}

\author{
Juliana de Oliveira \\ Escola de Engenharia, Arquitetura \\ Tecnologia \\ Universidade Anhembi Morumbi \\ São Paulo, SP - Brasil \\ jdoliveira.jdo@gmail.com
}

\author{
André Luiz Maciel Santana \\ Escola de Engenharia, Arquitetura e \\ Tecnologia \\ Universidade Anhembi Morumbi \\ São Paulo, SP - Brasil \\ andre.santana@anhembi.br
}

\author{
Vitor Matheus Ferraz Pereira \\ Escola de Engenharia, Arquitetura e \\ Tecnologia \\ Universidade Anhembi Morumbi \\ São Paulo, SP - Brasil \\ vmatheushs@gmail.com
}

\begin{abstract}
The studies and applications of nanofibers have grown over the years. It was observed that the properties of the nanometerscale yarns present advantages in applications in several areas, such as biomedical, energy storage and production, and applications involving water filtration. These materials are synthesized through a technical process and, for that reason, they are subject to the presentation of failures. The most common flaws are the formation of granules and pores. With the evolution of computing, applications that use machine learning resources can assist in detecting these failures. This work aims to evaluate and compare two different approaches to morphological analysis to see losses in nanofibers. Firstly, a data set was created using a Scanning Electron Microscope. After that, each image was analyzed by Image J software and by RNA solution. As a hypothesis, the article will assess whether the beads identification and the number of beads by the analog method are statistically similar (H0) or statistically different (H1) from the machine learning method. The preliminary results indicate that for the group that used 100 images and computer visualization, the analog method's accuracy was $7.23 \%$. In order to accuracy increase, another test with 150 more distinct images was done, bringing a new result of $55.09 \%$. The analysis time was considerably less when performed by the computational method. It was possible to conclude that the computational approach does not have the beads identification statistically similar to the analog way concerning the methodology used. Therefore, rejected HO. However, the directly proportional relationship of accuracy with the number of samples suggests that training with more various images can calibrate the algorithm.
\end{abstract}

\section{KEYWORDS}

Nanofiber, Machine Learning, Computer Vision, Beads.

\section{INTRODUÇÃo}

Nanofibras, definidas como fibras com o diâmetro, ou dimensional externo, de 1-100 nm e fibras submicrométricas 101-999 nm [1, 2], são desejadas para uma série de aplicações, incluindo biomédicas [3, 4], eletrônicas [8], ópticas [9], de filtros $[5,6,7]$ e de tecidos protetores. Atualmente existem trabalhos que reportam a eficiência do uso de nanofibras em combinação com nanopartículas em aplicações de filtros para microrganismos [10]. Entretanto, defeitos como poros e beads limitam a aplicação de nanofibras e conhecer o tipo, número e volume dos defeitos em nanofibras é fundamental no direcionamento dessa classe de materiais. Com o avanço da Inteligência Artificial e seu uso em reconhecimento de imagens, essa alternativa se apresenta como uma opção para caracterizar nanomateriais. Em função do exposto o objetivo desse trabalho é utilizar visão computacional para caracterizar defeitos em nanofibras.

\section{REVISÃO DA LITERATURA}

Além de conhecer a propriedade do material selecionado para a síntese das nanofibras, é de grande importância verificar se a nanofibra obtida após o processamento atingiu o resultado esperado, a fim de assegurar que ela está apta para aplicação [4-6]. Essa verificação geralmente é feita através de 
equipamentos de alta tecnologia, como o Microscópio Eletrônico de Varredura, comumente conhecido como MEV.

\section{SOLUÇÃO PROPOSTA}

Na etapa de análise das imagens e classificação dos dados coletados, foi observado a necessidade de otimização do tempo utilizado. Neste estágio, a extração destes dados é feita de maneira criteriosa e analítica por um profissional que disponibilizará do seu olhar treinado, para que a base de informações obtida seja satisfatória.

Visando minimizar os problemas gerados por esta situação, o estudo da Inteligência Artificial e de sua subdisciplina Aprendizagem de Máquina, mostraram-se potencialmente eficazes atuando neste tipo de obstáculo, onde programas de computador (algoritmos) aprendem associações de poder preditivo a partir de exemplos em dados [12-14].

Neste contexto, visa-se implementar as ferramentas necessárias para classificar analiticamente um determinado conjunto de dados.

Para a validação dos resultados, esse estudo levou em consideração três maneiras. A primeira delas foi obtida por meio do cálculo da acurácia, ou seja, a razão entre o número de beads identificados pela programação (bip) e a quantidade real de beads na imagem (identificadas manualmente, bir) o resultado dessa divisão foi multiplicado por 100. Definindo assim, o percentual de acurácia do programa conforme a Equação 1.

$$
\text { Acurácia }(\%)=\frac{\text { bip }}{\text { bir }} * 100
$$

Portanto, comparando o número de beads encontrados pela análise computacional com o número encontrado pela análise analógica, o valor obtido de acurácia foi de 7,23\%.

Acredita-se que a razão desse baixo percentual em precisão se deve por algumas razões relacionadas às imagens propriamente ditas, sendo elas a nitidez das imagens ou o contraste entre as cores. Esses pontos podem ter influenciado no baixo desempenho de reconhecimento de beads por uma limitação do software quando esses parâmetros não são claros nas figuras. Outra hipótese está relacionada a divisão feita entre as imagens selecionadas para treino e as imagens escolhidas para validação, podendo a escolha das amostras ou mesmo a quantidade de imagens ter interferido no reconhecimento. Sabe-se que quanto maior a quantidade de treinamentos com imagens diversas, maior será a chance de o algoritmo ser preciso. Além disso, caso as imagens da validação tenham configurações como luminosidade, nitidez ou contraste muito diferentes das condições das imagens do treinamento, pode-se obter também um resultado abaixo do esperado [24]. Levando esses pontos em consideração, o grupo decidiu realizar um novo treinamento agora com 150 imagens, ou seja, com quantidade um pouco maior do que a anterior e imagens mais diversificadas entre si. Com esse novo treinamento, obteve-se uma acurácia de 55,09\%.

Contudo, como segundo método de validação, foi comparado o tempo de análise via Aprendizado de Máquina e pesquisadores, que marcando o tempo de análise de suas amostras com um cronometro, registraram os resultados em uma tabela.

Dessa forma, vê-se os resultados de tempo obtidos conforme a Tabela I.

\begin{tabular}{cc}
\hline $\begin{array}{c}\text { Analógico } \\
\text { (Image) }\end{array}$ & $\begin{array}{c}\text { Computacional } \\
\text { (Algoritmo adaptado) }\end{array}$ \\
\hline $01: 23: 34$ & $00: 00: 51$ \\
\hline
\end{tabular}

Table 1. Comparação do tempo gasto entre o método Analógico e método Computacional.

Como última forma de validação dos resultados, o teste estatístico realizado demonstrou que o método analógico apresenta uma maior variação no processo de contagem de beads. Conforme pode ser visto na Figra 1, o método analógico apresentou uma maior variação que o método computacional.

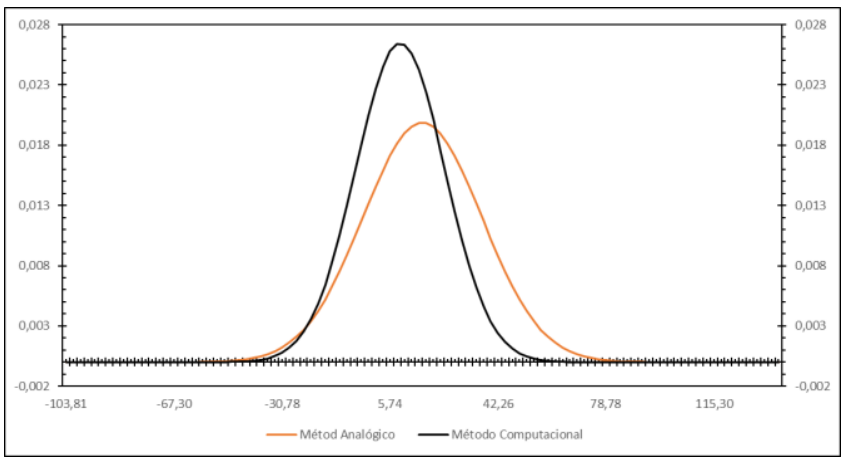

Figure 1. Comparação entre o método analógico e mótodo computacional

Com base nos resultados obtidos, foi possível concluir que o método computacional possui uma eficiência superior no que compete ao tempo gasto para análise de imagens por ser mais rápido. No entanto, existe uma possibilidade de melhoria no que se compete a acurácia do método computacional, através de mais treinamentos com uma maior diversidade de imagens.

\section{CONSIDERAÇÕES FINAIS}

Por fim, foi possível atender o objetivo esperado comprovando que a análise pelo método computacional é mais eficiente em termos do tempo gasto quando comparado ao método analógico, entretanto a acurácia da programação é um ponto a ser desenvolvido.

Dessa forma, foi feito um troubleshooting e entende-se que a qualidade e quantidade das imagens podem ter sido causasraízes para o problema inicial de baixa precisão bem como a quantidade de treinamentos. 
Assim, sugere-se que para próximos estudos, os pontos acima destacados como hipóteses para a causa raiz da baixa acurácia sejam levados em consideração na escolha de amostras para novos treinamentos.

\section{REFERÊNCIAS}

[1] COZMUTA, I.; BLANCO, M.; GODDARD, W. A., III. Gas sorption and barrier properties of polymeric membranes from molecular dynamics and Monte Carlo simulations. Abstracts of Papers of the American Chemical Society, v. 234, Aug 192007.

[2] VALIPOURI, A. et al. Experimental and numerical study on isolated and nonisolated jet behavior through centrifuge spinning system. International Journal of Multiphase Flow, v. 69, n. 0, p. 93-101, 3// 2015.

[3] WANG, L. et al. Fabrication of polymer fiber scaffolds by centrifugal spinning for cell culture studies. Microelectronic Engineering, v. 88, n. 8, p. 17181721, Aug 2011.

[4] YILDIRIMER, L.; SEIFALIAN, A. M. Three-dimensional biomaterial degradation - Material choice, design and extrinsic factor considerations. Biotechnology Advances, v. 32, n. 5, p. 984-999, Sep-Oct 2014.

[5] LEUNG, W. W.-F.; HUNG, C.-H.; YUEN, P.-T. Effect of face velocity, nanofiber packing density and thickness on filtration performance of filters with nanofibers coated on a substrate. Separation and Purification Technology, v. 71 , n. 1 , p. $30-37,1 / 29 / 2010$

[6] CHO, D. et al. Further improvement of air filtration efficiency of cellulose filters coated with nanofibers via inclusion of electrostatically active nanoparticles. Polymer, v. 54, n. 9, p. 2364-2372, 4/19/ 2013.

[7] BAHNASI, F. I.; RAHMAN, A. N. A. A.; ABU-HASSAN, M. I. The impact of recycling and repeated recycling on shear bond strength of stainless-steel orthodontic brackets. Orthodontic Waves, v. 72, n. 1, p. 16-22, 2013.

[8] BAHNASI, F. I.; RAHMAN, A. N. A. A.; ABU-HASSAN, M. I. The impact of recycling and repeated recycling on shear bond strength of stainless-steel orthodontic brackets. Orthodontic Waves, v. 72, n. 1, p. 16-22, 2013.

[9] GIBSON, P. W. et al. Application of Nanofiber Technology to Nonwoven Thermal Insulation. Journal of Engineered Fibers and Fabrics, v. 2, n. 2, p. 32 40, 20072007.

[10] BERGSHOEF, M. M.; VANCSO, G. J. Transparent nanocomposites with ultrathin, electrospun nylon-4,6 fiber reinforcement. Advanced Materials, v. 11, n. 16, p. 1362-1365, Nov 101999.

[11] Borkow G, Zhou SS, Page T, Gabbay J. A novel anti-influenza copper oxide containing respiratory face mask. PLoS One. 2010 Jun 25;5(6):e11295.

[12] DO MÍNGUEZ, C., HERAS, J., PASCUAL, V. IJ-OpenCV: Combining ImageJ and OpenCV for processing images in biomedicine. Computers in Biology and Medicine, v.84, p. 189-194. May 1, 2017.

[13] WIDODO, C. E., ADI, K., GERNOWO, R. Medical image processing using python and open cv. IOP Publishing Ltd. Journal of Physics: Conference Series, v. 1524. October 22, 2019.

[14] Trishan Panch, Peter Szolovits and Rifat Atun Artificial intelligence, machine learning and health systems. 2018. October. 